\title{
VISCOUS-FLOW CALCULATIONS FOR MODEL AND FULL-SCALE CURRENT LOADS ON TYPICAL OFFSHORE STRUCTURES
}

\author{
A.H. KOOP* , C.M. KLAIJ" ${ }^{\#}$ AND G. VAZ\# \\ *Corresponding author, email: a.koop@marin.nl \\ *\# Maritime Research Institute Netherlands (MARIN) \\ P.O. Box 28 \\ 6700 AA Wageningen, The Netherlands \\ web page: http://www.marin.nl
}

Key words: CFD, RANS, Current Loads, Verification, Validation, Scale Effects, LNG Carrier, SemiSubmersible

\begin{abstract}
In this paper, CFD calculations for current loads on an LNG carrier and a semi-submersible are presented, both for model and full-scale situations, for current angles ranging from 180 to 0 degrees. MARIN's in-house URANS code ReFRESCO is used. Numerical studies are carried out concerning iterative convergence and grid refinement. In total, more than 100 calculations have been performed. Detailed verification analysis is carried out using modern techniques, and numerical uncertainties are calculated. Afterwards, quantitative validation for model-scale Reynolds number is done taking into account numerical and experimental uncertainties. Scale effects on the current coefficients are investigated, having in mind the estimated numerical uncertainties, and unsteady effects are briefly studied. Good iterative convergence is obtained in most calculations, i.e. a decrease in residuals of more than 5 orders is achieved. The sensitivity to grid resolution has been investigated for both model and full scale using five consecutively refined grids and for 3 current headings. The differences in the solution between two consecutive refinements converge for all cases. The numerical uncertainties are larger for angles with small values of the loads. Comparison with experiments shows that ReFRESCO provides good quantitative prediction of the current loads at model scale: for angles with larger forces the CFD results are validated with $15 \%$ of uncertainty. To determine scale effects the numerical uncertainties must be considered in order to prevent wrong conclusions drawn on basis of numerical differences rather than on physical differences. For the full-scale results larger numerical uncertainties are found than for model scale and for absolute values for scale effects this uncertainty should be improved. For the LNG carrier significant scale effects, i.e. more than $40 \%$, have been obtained for current angles where the friction component is dominant. For these cases the numerical uncertainty is relatively low. For the other current angles differences of $8-30 \%$ between model and full scale can be observed, but here larger numerical uncertainties are found. For the semi-submersible the numerical uncertainties for the full-scale results are larger than for the LNG carrier. For the semi-submersible the pressure component of the force is highly dominant, i.e. larger than $90 \%$ of the total force. On average the full-scale current coefficients are $20 \%$ lower than at model scale, but larger differences for a number of angles can be observed.
\end{abstract}

\section{INTRODUCTION}

At present, most of the design of offshore structures is done based on current loads coming from empirical methods or from model-scale experiments. These are usually conservative and therefore adequate in this phase. However, there are some counter arguments to this reasoning: 1) for example, a fixed cylinder experiences higher loads at super-critical Reynolds numbers than in the drag-crisis at lower Reynolds numbers. Or, as observed in the Current Affairs JIP [1], full-scale average forces on a schematic semi-submersible are lower than at model scale, but both frequency and amplitudes of full-scale loads are larger; 2) if one can improve the accuracy of the loads used in the design phase, the safety margins can 
be reduced decreasing the manufacturing costs of the structures and improving the dynamic-positioning capabilities. Therefore, there is a real need for full-scale experiments, full-scale calculations or (general) scaling rules. However, full-scale experiments are scarce, difficult to design and to carry out, and when performed kept confidential. General scaling rules such as used for ship resistance are not easily devised for these kind of complex flows. Thus, currently one is left to full-scale CFD calculations.

Nowadays, most engineers, including the authors, perform CFD at model scale and in steady mode to calculate current coefficients on offshore constructions. Again, the usual reasoning is that scale effects are small for this type of structures, that model-scale calculations are necessary for validation anyhow, and that unsteady calculations are not needed and/or too expensive. Moreover, usually there is no time/money to perform thorough numerical sensitivity variations, to achieve sufficient iterative convergence, and to perform verification studies.

The major objectives of this paper are then fourfold: 1) perform detailed verification studies for model and full-scale calculations of current loads, on two typical offshore structures, for several current angles from 180 to 0 degrees; 2) validate the model-scale numerical results with experimental data; 3) study the scale effects on the current loads; 4) perform a preliminary study on possible unsteady effects on model and full-scale loads.

Modern verification and validation techniques [2] are used in order to quantitatively asses numerical, experimental and validation uncertainties. Without those, the accuracy of the numerical results cannot be determined and conclusions on scale effects cannot be drawn. However, this requires many calculations and in total more than 100 calculations have been performed.

An LNG carrier appended with bilge-keels and rudder (streamlined body), and a semi-submersible constituted by four rounded-square columns mounted on large block-coefficient ship-shaped pontoons (blunt body) are considered, since they are typical offshore constructions. MARIN's in-house URANS code ReFRESCO [3] is used. Figure 1 presents the geometries and illustrates the calculated flow field for a specific current angle. Previous work done on these structures $[1,4,5]$ is here extended, and the lessons learned from the Current Affairs JIP, see [1, 4], are considered in order to improve the accuracy of the results.

The paper is organized as follows. After this introduction, definitions and details on the structures and measurements are presented, followed by the numerical settings used for the calculations. Afterwards, and for both the LNG carrier and semi-submersible, the iterative convergence and numerical uncertainty is discussed followed by detailed validation, and study of scale effects. Additionally, preliminary studies on unsteady effects are shown for the semi-submersible. Finally, major conclusions and further work are presented.

\section{DEFINITIONS}

The reference frame and current coefficients are defined following the OCIMF [6] convention, see Figure 2: the $x$-axis points towards the bow, the $y$-axis points towards portside meaning that 180 degrees corresponds to head-on current, 135 degrees to bow-quartering current and 90 degrees to beam-on current.

The definitions of the force and moment coefficients are given in Table 1. The Reynolds number and Froude number are:

$$
\mathrm{Re}=\frac{\rho U_{\mathrm{ref}} L_{\mathrm{ref}}}{\mu}, \quad \operatorname{Fr}=\frac{U_{\mathrm{ref}}}{\sqrt{g L_{\mathrm{ref}}}} .
$$

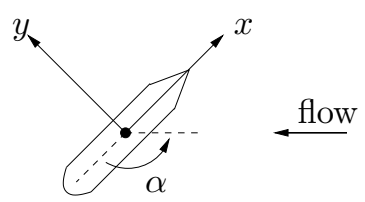

Figure 2: Reference frame

The dimensionless current coefficients are:

$$
C_{X, Y}=\frac{F_{X, Y}}{\frac{1}{2} \rho U_{\mathrm{ref}}^{2} L_{\mathrm{ref}} T}, \quad C_{M}=\frac{M_{Z}}{\frac{1}{2} \rho U_{\mathrm{ref}}^{2} L_{\mathrm{ref}}^{2} T} .
$$

For the LNG carrier the reference length $L_{\text {ref }}$ is chosen equal to $L_{\mathrm{pp}}$. For confidentiality reasons the force coefficients for the semi-submersible are scaled by the maximum value found in the wind tunnel experiments. In Section 6 these scaled values are denoted by $C_{X}^{*}, C_{Y}^{*}$ and $C_{M}^{*}$. 


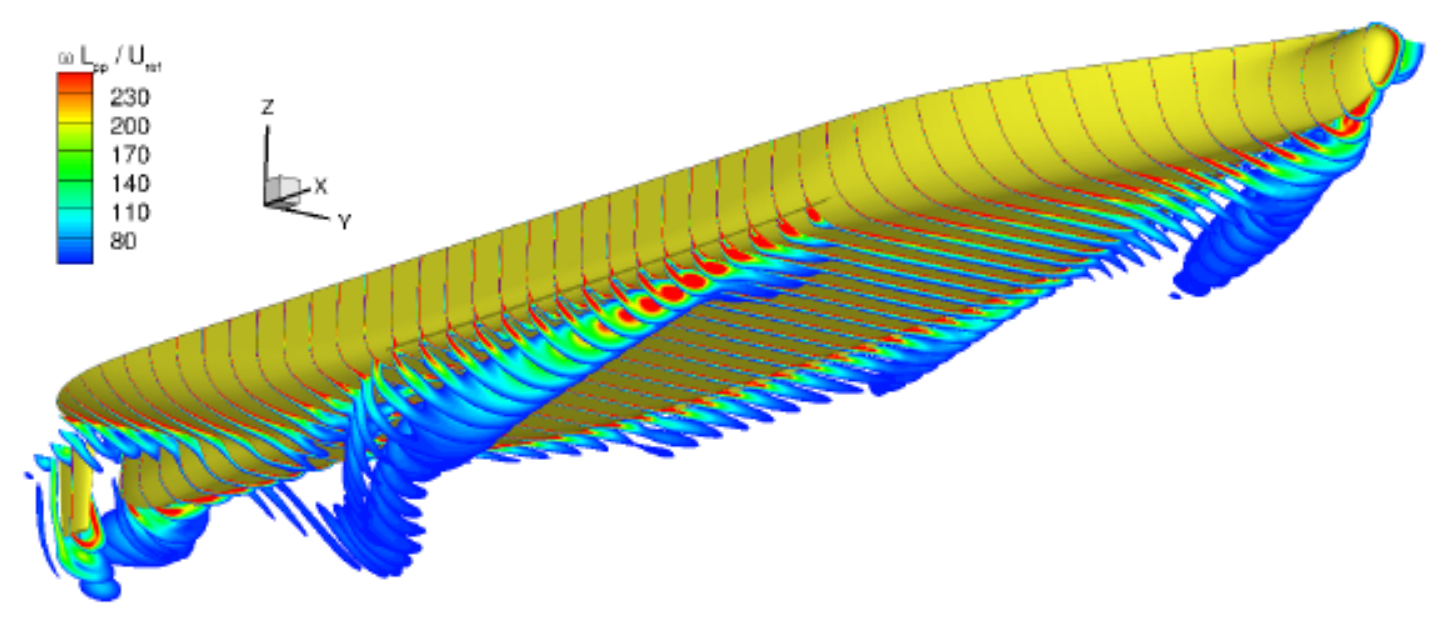

(a) LNG carrier at 140 degrees current heading.

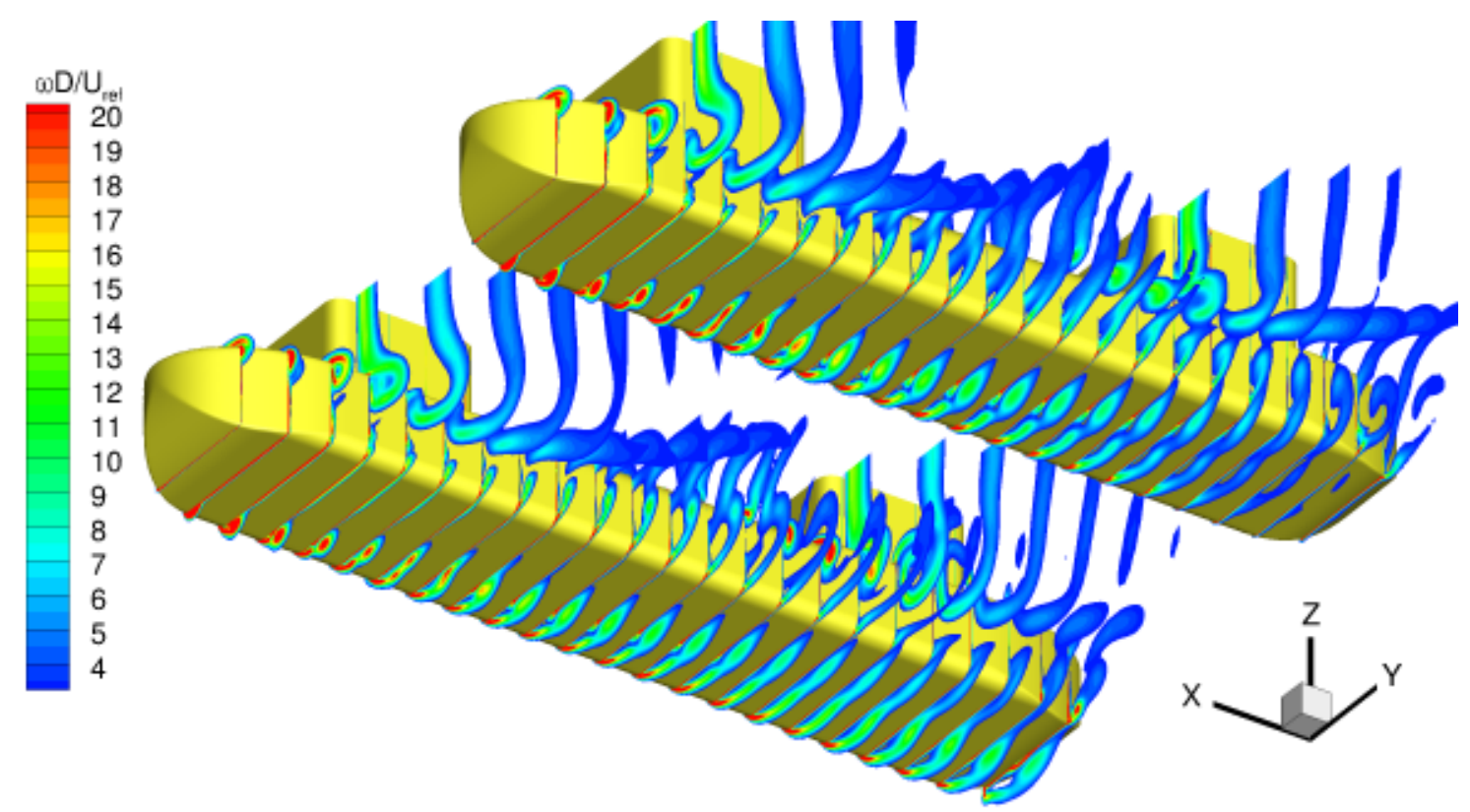

(b) Semi-submersible at 150 degrees current heading.

Figure 1: Impression of the flow field around the LNG carrier and semi-submersible illustrated by the vorticity distribution around the structures.

Table 1: Nomenclature.

\begin{tabular}{llll}
\hline$\alpha$ & current angle $[\mathrm{deg}]$ & $F=\left(F_{X}, F_{Y}, F_{Z}\right)$ & forces $\left[\mathrm{kg} \cdot \mathrm{m} \cdot \mathrm{s}^{-2}\right]$ \\
$L_{\mathrm{pp}}$ & length between perpendiculars $[\mathrm{m}]$ & $M=\left(M_{X}, M_{Y}, M_{Z}\right)$ & moments $\left[\mathrm{kg} \cdot \mathrm{m}^{2} \cdot \mathrm{s}^{-2}\right]$ \\
$T$ & draft $[\mathrm{m}]$ & $\rho$ & density $\left[\mathrm{kg} \cdot \mathrm{m}^{-3}\right]$ \\
$W_{D}$ & water depth $[\mathrm{m}]$ & $\mu$ & dynamic viscosity $\left[\mathrm{kg} \cdot \mathrm{m}^{-1} \cdot \mathrm{s}^{-1}\right]$ \\
$U_{\text {ref }}$ & reference velocity $\left[\mathrm{m} \cdot \mathrm{s}^{-1}\right]$ & $\vec{\omega}=\nabla \times \vec{u}$ & vorticity vector $\left[\mathrm{s}^{-1}\right]$ \\
$L_{\mathrm{ref}}$ & reference length $[\mathrm{m}]$ & $\omega=|\vec{\omega}|$ & norm of the vorticity vector $\left[\mathrm{s}^{-1}\right]$ \\
$T_{\text {ref }}=L_{\mathrm{ref}} / U_{\text {ref }}$ & reference time $[\mathrm{s}]$ & & \\
\hline
\end{tabular}




\section{MEASUREMENTS}

\subsection{LNG Carrier}

During the HAWAII JIP, current loads for a 135,000 $\mathrm{m}^{3}$ LNG carrier have been measured in MARIN's shallow water basin by towing the model through otherwise calm water for flow angles between 0 and 180 degrees, see [7]. The model of the LNG carrier included bilge keels, propeller and rudder and the scale was 1:50. Studs were used on the bow and stern to trigger the boundary layer to become turbulent. From a Reynolds sensitivity check it was concluded that for the tested current veloctiy, the Reynolds dependency at model scale on the measured force coefficients was less than $5 \%$. The ratio of the water depth to the draft is $W_{D} / T=4.8$, indicating that shallow-water effects might have an influence on the current forces, see also [4]. In order for the free-surface effects to be negligible the Froude number was set small to Fr $=0.04$. During the experiments no significant waves were observed, see [7]. The model-scale Reynolds number is equal to $1.6 \cdot 10^{6}$. At full scale the Reynolds number is equal to $5 \cdot 10^{8}$. The full-scale particulars of the LNG carrier are given in Table 2.

Table 2: Main particulars of $135,000 \mathrm{~m}^{3}$ LNG carrier

\begin{tabular}{ll}
\hline Description & Value \\
\hline Length between perpendiculars & $L_{\mathrm{pp}}=274.0 \mathrm{~m}$ \\
Draft & $T=11.0 \mathrm{~m}$ \\
Breadth & $B=44.2 \mathrm{~m}$ \\
Water-depth & $W_{D}=53.0 \mathrm{~m}$ \\
Capacity & $135,000 \mathrm{~m}^{3}$ \\
Current velocity & $U_{\text {ref }}=2.06 \mathrm{~m} / \mathrm{s}$ \\
\hline
\end{tabular}

\subsection{Semi-submersible}

Wind-tunnel tests at Force Technology [8] have been carried out at scale 1:200. The current loads have been tested in an airflow corresponding to a vertically uniform current. The forces and moments were measured for angles in the range 0 to 360 degrees in increments of 10 degrees. The tests have been carried out with 8 thrusters placed under the pontoons modeled by a single ring. No roughness was applied on the hull. Later in the project the semi-submersible was also tested in MARIN's Offshore Basin and the length of the pontoons has been changed between the wind tunnel tests and the basin measurements. Therefore, the length of the pontoons for the wind tunnel tests was $4.5 \%$ shorter than used in the Offshore Basin and CFD calculations. The comparison between the basin measurements, wind tunnel and CFD results can be found in [5].

The Froude number based on the column diameter at water surface level is equal to $\mathrm{Fr}=0.11$. The Reynolds number based on $L_{\mathrm{pp}}$ is equal to $2 \cdot 10^{8}$ at full scale and equal to $5 \cdot 10^{5}$ in the wind tunnel and model-scale CFD calculations. For confidentiality reasons the main particulars of the semi-submersible can not be shared in this paper.

\section{COMPUTATIONAL SETUP}

\subsection{Computational grids}

For both geometries five consecutively refined block-structured grids have been constructed using the package GridPro [9], see Table 3. At model scale the maximum $y^{+}$value is below 1 on all grids and no wall functions are used. However, for the LNG carrier at full scale, having $y^{+}$values below 1 requires extremely thin cells, especially close to the bilge keels, which lead to severe numerical problems. Using the same clustering as on model scale, we obtain maximum $y^{+}$values below 100 and wall functions are applied. For the semi-submersible the model-scale grids are adapted by refining the first element in the boundary layer to obtain maximum $y^{+}$values below 11 and no wall functions are used.

Wall functions model the viscous sublayer near the wall. The use of wall functions effectively avoids numerical issues due to extremely thin cells but also introduces an additional modelling error in the computations. The effect of using wall functions is not addressed in this paper, but certainly deserves further investigation. 

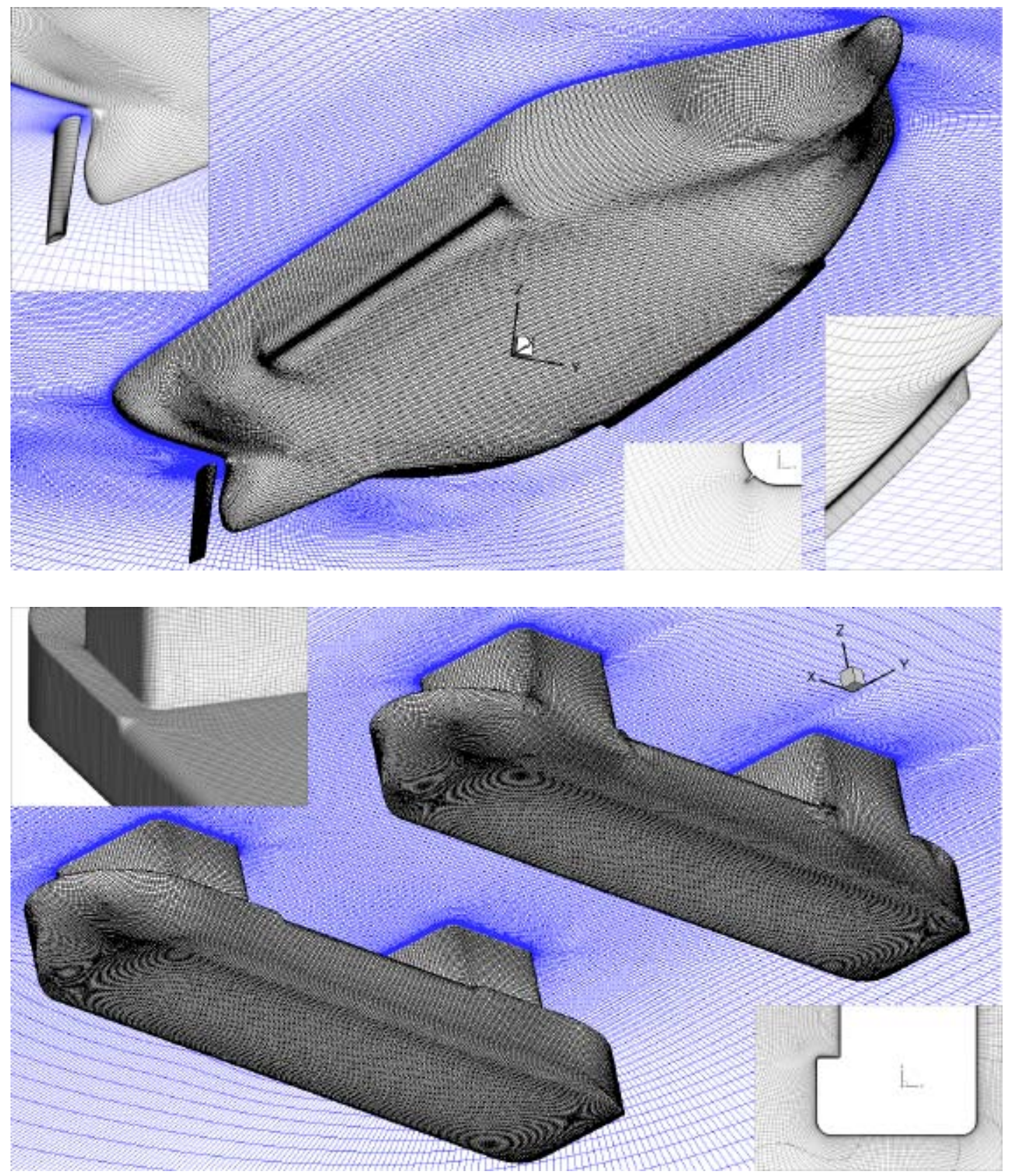

Figure 3: Computational grid for the LNG carrier and semi-submersible. The black lines denote the grid on the surface of the carrier and the blue lines denote the grid on the water surface.

Table 3: Information on computational grids for the LNG carrier and semi-submersible.

\begin{tabular}{|c|ccc|cc|cc|}
\hline & \multicolumn{3}{|c|}{ LNG carrier } & \multicolumn{3}{c|}{ semi-submersible } \\
\cline { 2 - 8 } & \multicolumn{3}{|c|}{ model scale } & full scale & \multicolumn{2}{c|}{ model scale } & \multicolumn{2}{c|}{ full scale } \\
& \# cells & $\max y^{+}$ & $\max y^{+}$ & \# cells & $\max y^{+}$ & \# cells & $\max y^{+}$ \\
\hline very coarse & $0.70 \mathrm{M}$ & 0.95 & 99.5 & $0.9 \mathrm{M}$ & 1.1 & $1.2 \mathrm{M}$ & 12 \\
coarse & $1.15 \mathrm{M}$ & 0.77 & 78.5 & $1.7 \mathrm{M}$ & 0.81 & $2.4 \mathrm{M}$ & 9.0 \\
medium & $1.85 \mathrm{M}$ & 0.74 & 74.8 & $3.4 \mathrm{M}$ & 0.62 & $4.7 \mathrm{M}$ & 5.8 \\
fine & $3.33 \mathrm{M}$ & 0.59 & 68.1 & $7.0 \mathrm{M}$ & 0.53 & $9.5 \mathrm{M}$ & 5.0 \\
very fine & $5.72 \mathrm{M}$ & 0.58 & 61.5 & $14.0 \mathrm{M}$ & 0.36 & $19.3 \mathrm{M}$ & 4.2 \\
\hline
\end{tabular}




\subsection{Boundary and initial conditions}

Since the Froude number is very small, wave generation is neglected and a symmetry boundary condition is imposed on the water surface. The bottom surface of the domain is positioned at the same depth as in the measurements. For the LNG carrier the water depth to draft ratio is equal to 4.8. In [4] the effect of the distance of the bottom surface has been investigated and it was concluded that for this ratio the bottom surface should be taken into account. Therefore, a free-slip wall condition is prescribed at the bottom surface. For the semi-submersible the water depth to draft ratio is more than 25 , so a constant-pressure boundary condition is prescribed.

In [4], it was shown that the blockage effect of the basin side walls is negligible for the LNG carrier. Therefore, a cylindrical domain is chosen in order to use the same grid for all current angles. The cylinder is centered at the origin and has radius $3.5 L_{\mathrm{pp}}$ for the $\mathrm{LNG}$ carrier and $4 L_{\mathrm{pp}}$ for the semi-submersible. At the cylindrical boundary a constant uniform velocity is prescribed corresponding with the current angle, together with the eddy-viscosity to laminar-viscosity ratio and the turbulence intensity. For model scale the turbulence intensity is chosen equal to $1 \%$ and the eddy-viscosity ratio is set to 1.0. For full scale these values are set to $10 \%$ and 100.0, respectively. At the outflow, Neumann boundary conditions are applied for all variables.

For the coarsest grids the initial conditions for the calculations are defined in each computational cell by setting the velocity equal to the constant uniform velocity of the inflow boundary, the pressure is chosen equal to the reference pressure at the outflow boundary and the turbulence intensity and eddyviscosity ratio equal to the inflow boundary settings. For the calculations on finer grids the solution on coarser grids is interpolated to the finer grid to serve as the initial condition. This procedure reduces computational time compared to calculations started from uniform flow.

\subsection{ReFRESCO}

The CFD calculations in this paper are carried out using MARIN's in-house viscous-flow URANS code ReFRESCO [3]. ReFRESCO is targeted and optimized for hydrodynamic applications exclusively, and it has already been applied to several typical offshore flows. In particular, current, wind and manoeuvring coefficients of semi-submersibles, submarines and ships have been successfully verified and validated, $[1,4,5,10,11]$. For all calculations here presented the following numerical settings have been used: 1) QUICK scheme for convection discretization of the momentum equations; 2) Central scheme for diffusion discretization; 3) Upwind scheme for convection discretization of the turbulence equations. The SST k- $\omega$ turbulence model [12] is used for all calculations. Parallelization has been employed because of the long computational times: some calculations have been carried out using 64 quad-core processors.

\subsection{Verification and validation procedures}

In any numerical calculation there are intrinsic errors which have to be controlled, and if possible quantified, e.g. iterative and discretization errors. However, for a complex CFD calculation this can be very time-consuming. Iterative errors are due to non-linear algorithms and iterative solvers utilized, and in principle should be of the same order as the round-off error. Previous studies with two different CFD codes, see [2], have shown that the iterative error should be at least two orders of magnitude lower than the discretization error, in order not to influence the accuracy of the results.

Several methodologies are available to determine the numerical uncertainty related to the discretization error $[13,14]$. In this paper we follow the approach as described in [2]. The numerical uncertainty $U_{\phi}$ for any arbitrary flow quantity $\phi$ is determined using $U_{\phi}=F_{s}|\epsilon|$, where $F_{s}$ represents a safety factor and $\epsilon$ denotes an estimate of the discretization error. These are determined by applying a least-squares fit of a error power law, $\alpha h_{i}^{p}$, to the results obtained for grids with different densities or relative step size $h_{i}$. The choice of error estimator and safety factor depends on the apparent convergence condition (monotonic, oscillatory, non-convergent) and apparent order of convergence $p$ (see for further details [2]).

Validation can only be done after verification and it involves numerical, experimental and parameter uncertainties. The aim of validation is to estimate the modelling error of a given mathematical model in relation to a given set of experimental data. If the validation is successful one cannot say that the code is validated, only that the model is valid for the problem at hand. A well-documented procedure [14] already applied for other ReFRESCO applications [11, 15, 16] is here employed. It compares the 
validation uncertainty $U_{v a l}$ with the validation comparison difference $E$, which are defined by

$$
U_{v a l}=\sqrt{U_{\phi}^{2}+U_{i n p}^{2}+U_{e x p}^{2}}, \quad E=\phi_{i}-\phi_{\text {exp }},
$$

with $U_{\phi}$ the numerical uncertainty, $U_{i n p}$ the parameter uncertainty, i.e. uncertainties in the fluid properties, geometry and boundary conditions, and $U_{\exp }$ the experimental uncertainty. $\phi_{i}$ and $\phi_{\text {exp }}$ represent the numerical and experimental value, respectively. The outcome of the validation exercise is decided from the comparison of $|E|$ with $U_{v a l}$ :

- If $|E|>U_{v a l}$, the comparison difference is probably dominated by the modelling error, which indicates that the model must be improved;

- If $|E|<U_{\text {val }}$, the modelling error is within the "noise level" imposed by the three uncertainties. This can mean two things: if $E$ is considered sufficiently small, the model and its solution are validated (with $U_{v a l}$ precision) against the given experiment; else the quality of the numerical solution and/or the experiment should be improved before conclusions can be drawn about the adequacy of the mathematical model.

For a precise validation, the experimental uncertainty $U_{\text {exp }}$ is also needed. This is rarely assessed, and few experimental data for current loads exists in the open-literature for which uncertainties are presented. In this paper, the experimental uncertainty $U_{\text {exp }}$ is assumed to be equal to $5 \%$ for the current loads obtained in MARIN's shallow-water basin and $10 \%$ for those from the wind tunnel. These values are based on in-house studies taking into account reproducibility for different test runs, manufacturing tolerances and uncertainties of the sensors. The experimental uncertainty for the wind-tunnel experimental data is larger due to the measurement procedure: the model is placed on a flat splitter plate to position the model in an uniform airflow. However, along the splitter plate a boundary layer develops which has an influence on the forces on the hull.

\section{RESULTS FOR LNG CARRIER}

\subsection{Iterative convergence}

The level and speed of the iterative convergence is dependent on the angle, grid resolution and scale of the calculation. Figure 4 shows three typical convergence histories: 1) for model scale and 180 degrees the convergence is fast, and a decrease in residuals more than 6 orders is obtained; 2) for model scale and 140 degrees the convergence is slower and the residuals stagnate at 5 orders of decrease; 3) for full scale at 90 degrees, the convergence history is the worst for all calculations presented in the current paper, and the residuals stagnate at 3-4 orders. For all cases here presented, the force coefficients become constant after a few hundred iterations and no oscillations are seen, not even for current angles 140 degrees and 90 degrees. In general, full-scale calculations are more difficult to converge than for model scale, and for the same inflow angle one order less of residuals decrease is obtained.

\subsection{Numerical uncertainties}

In Table 4 the current loads at model and full scale are presented for three headings using five consecutively refined grids, together with the numerical uncertainties for the finest grid. Figure 5 shows the results of the uncertainty procedure explained in Section 4.4, for three angles, both for model and full scale. For the 140 degrees model-scale case the convergence is not monotonic and the uncertainty procedure is not able to perform a fit to the error power law. Nevertheless, it is able to estimate an uncertainty value. One can see that the differences in the solution between two consecutive refinements are getting smaller for all cases, with already relatively small values for the medium grid. However, this has no relation with the uncertainties calculated, which can still be large.

For model scale the numerical uncertainties are relatively small. In general, the uncertainties are larger for the coefficients with lower absolute values. At full scale the uncertainties are larger. Also, at full scale, the use of wall-functions adds an additional modeling error which could explain why $C_{X}$ is more sensitive to grid density than at model scale. The largest sensitivity is found at 140 degrees where significant flow separation and recirculation contradict the assumptions underlying wall functions. Notice though, that the uncertainty value of $94.3 \%$ for the full-scale $C_{X}$ at 140 degrees, corresponds to a variation of $2 \times 10^{-3}$ in this coefficient, i.e. in absolute magnitude this uncertainty is not relevant. 

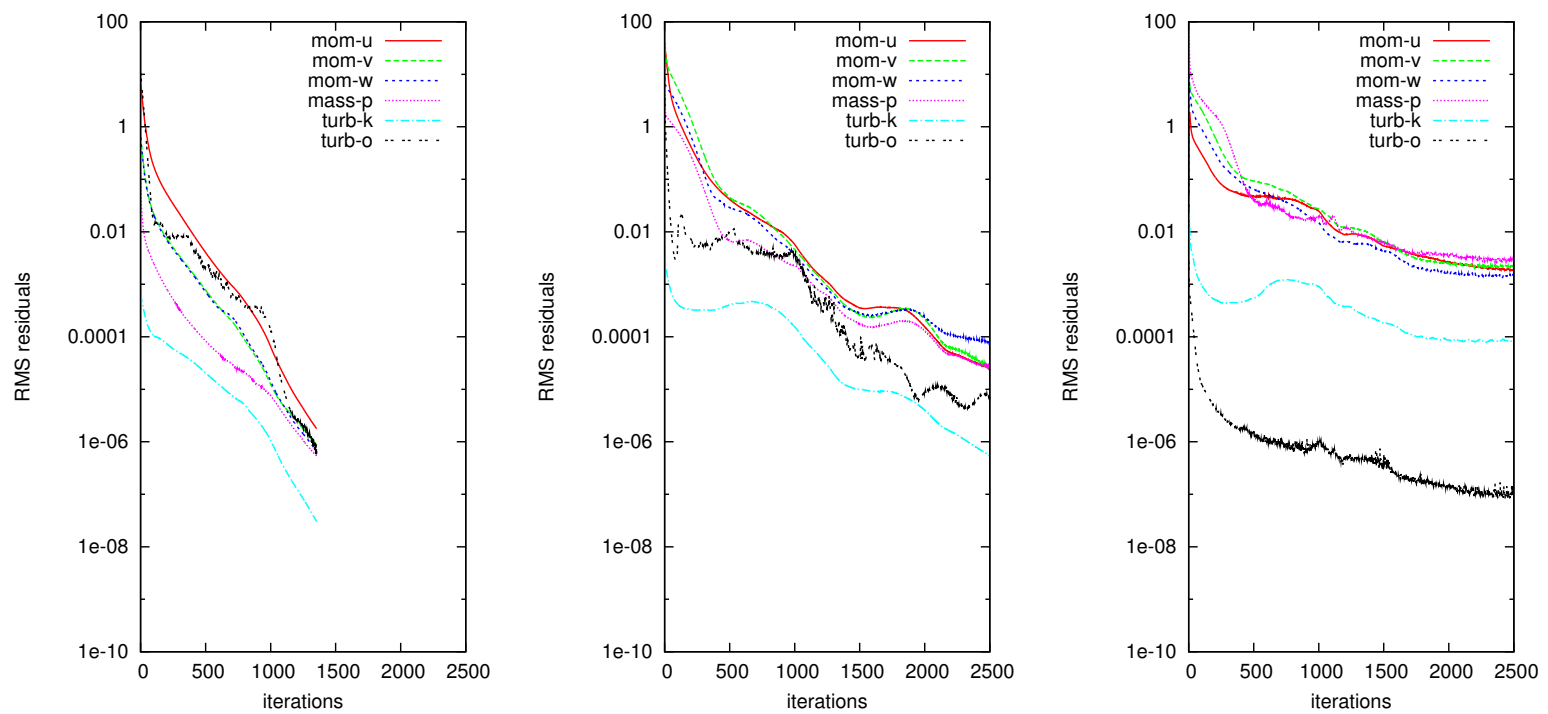

Figure 4: LNG carrier: examples of iterative convergence for model scale at 180 degrees (left); model scale at 140 degrees (middle); full scale at 90 degrees (right).
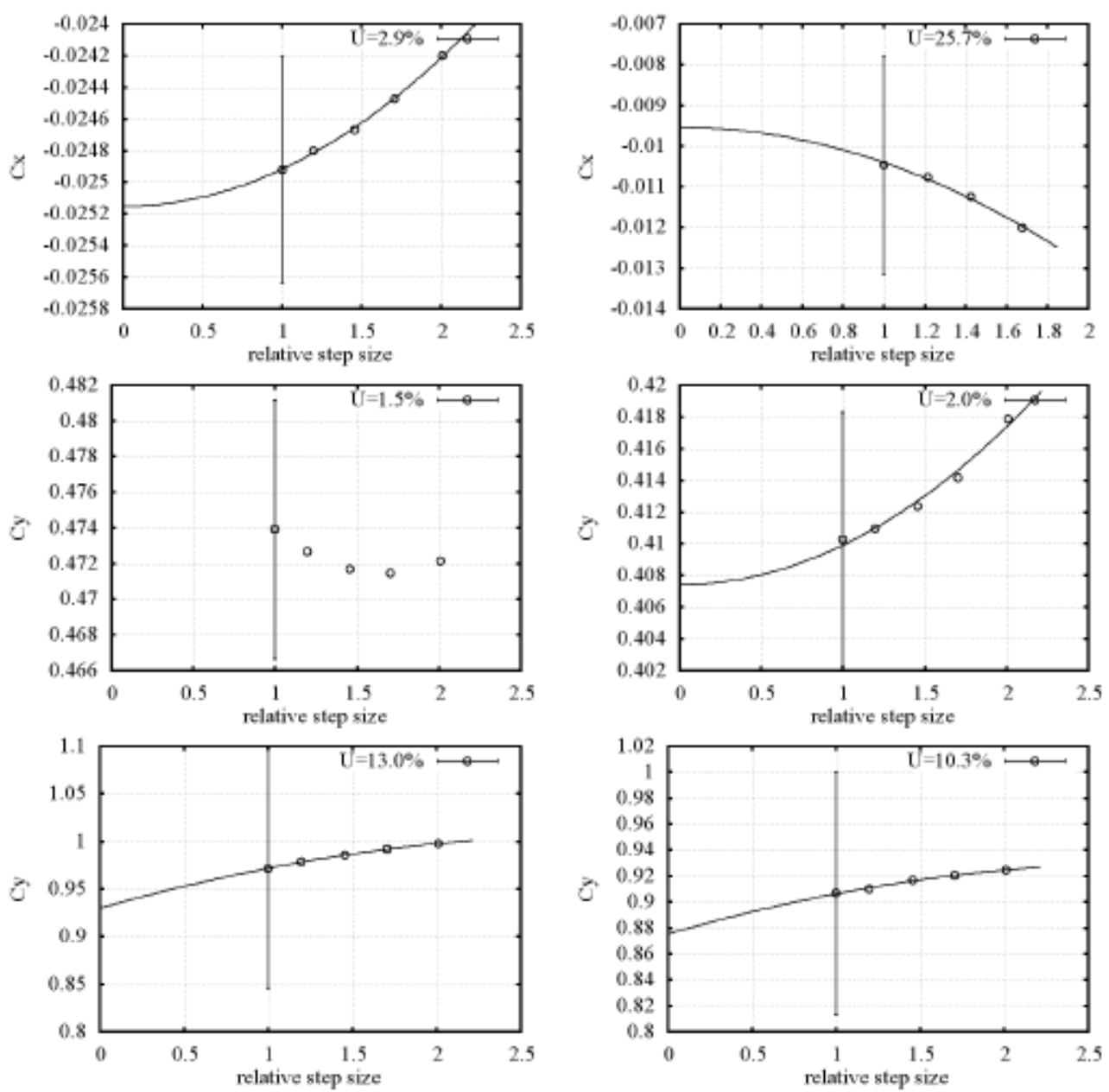

Figure 5: LNG carrier: examples of numerical uncertainty analysis for $C_{X}$ and $C_{Y}$ coefficient. Model scale (left); full scale (right); 180 degrees (top); 140 degrees (middle); 90 degrees (bottom). 
Table 4: LNG carrier: results for model and full-scale current coefficients at 180, 140 and 90 degrees obtained using grids with different resolution. Between brackets the difference compared to the result on the finest grid is presented. The numerical uncertainty $U_{\phi}$ for the results of each coefficient for the finest grid is also presented.

\begin{tabular}{|c|c|c|c|c|c|c|}
\hline & & 180 degrees & & 140 degrees & & 90 degrees \\
\hline & Grid & $C_{X}$ & $C_{X}$ & $C_{Y}$ & $C_{M}$ & $C_{Y}$ \\
\hline & very coarse & $-0.0241(-3.3 \%)$ & $-0.0137(-9.0 \%)$ & $0.4721(-0.4 \%)$ & $0.0749(+4.2 \%)$ & $0.9978(+2.7 \%)$ \\
\hline$\frac{0}{6}$ & coarse & $-0.0244(-2.1 \%)$ & $-0.0147(-13.1 \%)$ & $0.4714(-0.6 \%)$ & $0.0741(+3.1 \%)$ & $0.9916(+2.0 \%)$ \\
\hline-5 & medium & $-0.0246(-1.2 \%)$ & $-0.0156(-6.0 \%)$ & $0.4716(-0.5 \%)$ & $0.0732(+1.8 \%)$ & $0.9855(+1.4 \%)$ \\
\hline$\frac{0}{0}$ & fine & $-0.0248(-0.4 \%)$ & $-0.0163(-3.6 \%)$ & $0.4726(-0.3 \%)$ & $0.0719(0.0 \%)$ & $0.9781(+0.6 \%)$ \\
\hline$\stackrel{0}{z}$ & very fine & $-0.0249(0.0 \%)$ & $-0.0169(0.0 \%)$ & $0.4739(0.0 \%)$ & $0.0719(0.0 \%)$ & $0.9716(0.0 \%)$ \\
\hline & $U_{\phi}$ & $2.9 \%$ & $16.4 \%$ & $1.5 \%$ & $3.8 \%$ & $13.0 \%$ \\
\hline & very coarse & $-0.0120(-15.4 \%)$ & $0.00043(-90 \%)$ & $0.4178(+1.8 \%)$ & $0.0527(+4.4 \%)$ & $0.924(+1.9 \%)$ \\
\hline$\stackrel{0}{\pi}$ & coarse & $-0.0112(-7.7 \%)$ & $0.00200(-53.4 \%)$ & $0.4141(+0.9 \%)$ & $0.0520(+3.0 \%)$ & $0.920(+1.5 \%)$ \\
\hline ర్ల్ర & medium & $-0.0107(-2.9 \%)$ & $0.00312(-27.3 \%)$ & $0.4123(+0.5 \%)$ & $0.0519(+2.8 \%)$ & $0.916(+1.1 \%)$ \\
\hline$\equiv$ & fine & $-0.0104(0.0 \%)$ & $0.00394(-8.2 \%)$ & $0.4109(+0.1 \%)$ & $0.0515(+2.0 \%)$ & $0.910(+0.4 \%)$ \\
\hline 厌 & very fine & & $0.00429(0.0 \%)$ & $0.4102(0.0 \%)$ & $0.0505(0.0 \%)$ & $0.906(0.0 \%)$ \\
\hline & $U_{\phi}$ & $25.7 \%$ & $94.3 \%$ & $2.0 \%$ & $12.8 \%$ & $10.3 \%$ \\
\hline
\end{tabular}

\subsection{Validation with model-scale experiments}

From the verification exercise presented in Section 5.2 the numerical uncertainty $U_{\phi}$ is known. Having in the mind the experimental accuracy stated in Section 4.4, the validation procedure can be employed, and the comparison error $E$ and the validation uncertainty $U_{v a l}$ can be calculated. Note that, the numerical uncertainties have been only computed for the angles 180, 140 and 90 degrees. For the sake of the validation exercise we consider the maximum value of the uncertainties, for each coefficient, for the remaining angles. This will probably lead to larger validation uncertainties, but also to more validated results. For the LNG test-case alone around 40 calculations have been performed in total. In order to obtain $U_{\phi}$ for all angles and both in model and full-scale situations, more than 100 calculations should have been done. Table 5 shows the final results of the validation exercise. Most of the results for $C_{Y}$ are validated within $\pm 15 \%$ of uncertainty. It can be observed that for angles where the coefficients have small values the CFD results are not validated. The value for $C_{X}$ for 130 degrees shows the use of relative differences: the comparison difference $E$ is equal to $165 \%$, which is due to the fact that, coincidentally for this angle, the $C_{X}$ coefficient is almost zero.

Table 5: LNG carrier: comparison difference $E$ between model-scale CFD results and experimental values and validation uncertainty $U_{v a l}$. In green the validated results, in red the non-validated results.

\begin{tabular}{|c|c|cccccccccc|}
\hline & \multirow{2}{*}{ Coefficient } & \multicolumn{10}{|c|}{ Angle [deg] } \\
\cline { 3 - 12 } & & 180 & 170 & 160 & 150 & 140 & 130 & 120 & 110 & 100 & 90 \\
\hline \multirow{3}{*}{$E$} & $C_{X}$ & $-11 \%$ & $-11 \%$ & $-19 \%$ & $-25 \%$ & $+11 \%$ & $+165 \%$ & $+23 \%$ & $+40 \%$ & $+49 \%$ & - \\
& $C_{Y}$ & - & $-20 \%$ & $-8 \%$ & $-5 \%$ & $-8 \%$ & $-7 \%$ & $-4 \%$ & $+10 \%$ & $+11 \%$ & $+14 \%$ \\
& $C_{M}$ & - & $-7 \%$ & $+4 \%$ & $-4 \%$ & $-16 \%$ & $-21 \%$ & $-17 \%$ & $+0.5 \%$ & $+27 \%$ & - \\
\hline \multirow{3}{*}{$U_{\text {val }}$} & $C_{X}$ & $15 \%$ & $15 \%$ & $13 \%$ & $12 \%$ & $18 \%$ & $43 \%$ & $20 \%$ & $23 \%$ & $25 \%$ & - \\
& $C_{Y}$ & - & $10 \%$ & $12 \%$ & $12 \%$ & $12 \%$ & $12 \%$ & $12 \%$ & $14 \%$ & $14 \%$ & $15 \%$ \\
& $C_{M}$ & - & $4 \%$ & $4 \%$ & $4 \%$ & $4 \%$ & $3 \%$ & $3 \%$ & $4 \%$ & $5 \%$ & - \\
\hline
\end{tabular}

An extra outcome from the validation procedure is that the graphical comparison between numerical and experimental results can be done using error bars as presented in Figure 6. It can be observed that the global trend for the three coefficients is correctly captured by the CFD calculations for the complete range of current angles. Using this graphical presentation of the results, validation is obtained once the uncertainty-bars for both the experiments and numerical results overlap. 

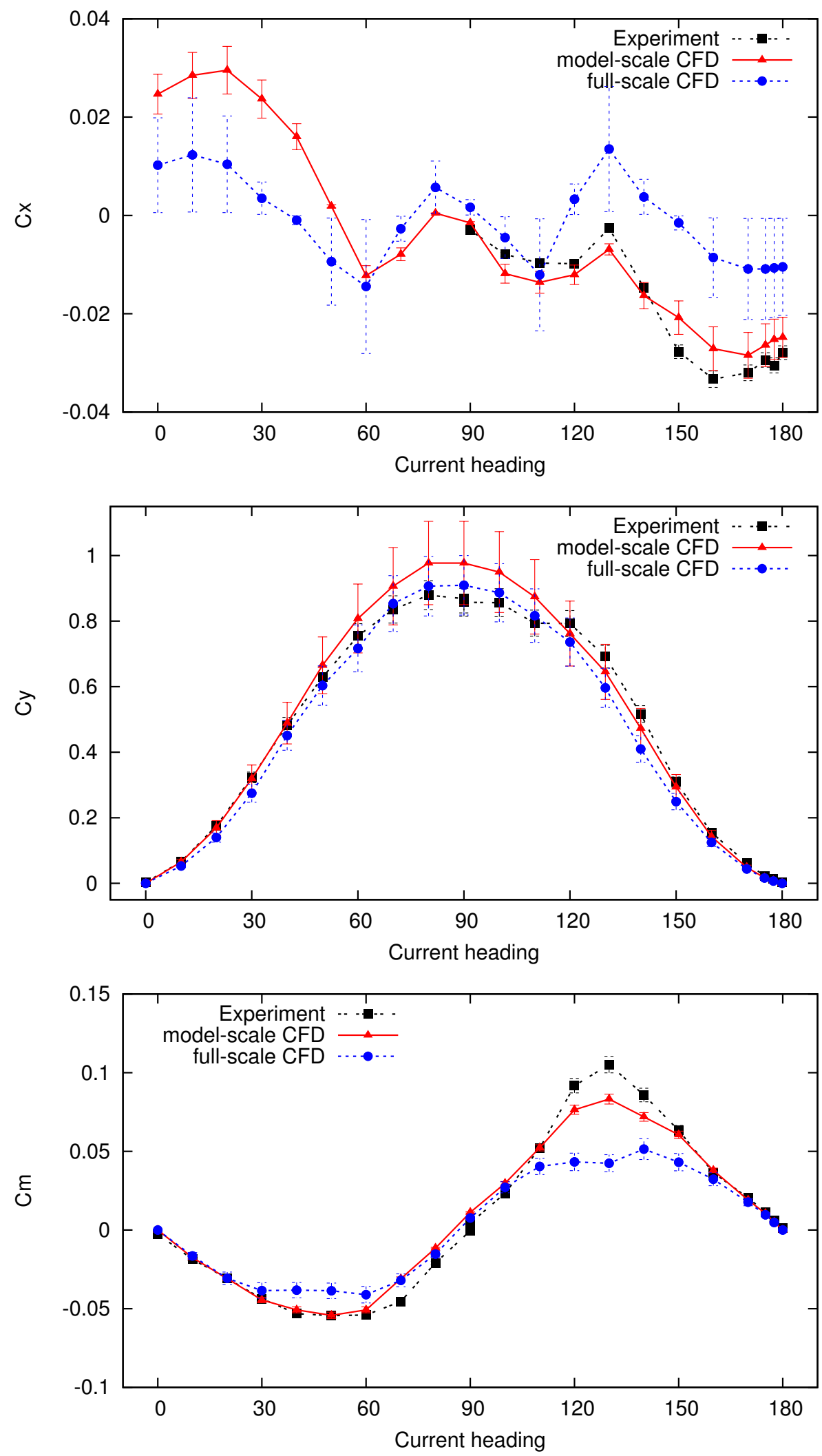

Figure 6: LNG carrier: model and full-scale current coefficients. CFD results versus experimental results. 


\subsection{Scale effects}

The full-scale results show that the force coefficients are typically lower than for model scale as illustrated in Figure 6 and Table 6. This means that the current loads at model scale, either obtained using CFD or from experiments, are conservative. In order to distinguish the real physical scale effects from possible numerical effects, a numerical comparison uncertainty has to be considered $U_{\text {comp }}=\sqrt{U_{\phi, M S}^{2}+U_{\phi, F S}^{2}}$. If the difference between model and full-scale results is larger than this comparison uncertainty then one can say that the scale effect has been captured correctly. If not, no conclusions must be made since the numerical errors taint the real physical effects. As presented in Table 6 the comparison uncertainties are too large for $C_{X}$ at 140 degrees and $C_{Y}$ at 90 degrees. Nevertheless, we can still, with some carefulness, observe several trends:

- Scale effects are largest when the friction component is dominant. For example, the $C_{X}$ coefficient at 180 degrees is $42 \% \pm 26 \%$ lower at full scale than at model scale;

- The scale effects for $C_{Y}$ are small. For $C_{X}$ the scale effects are larger. For $C_{M}$ it depends on the angle, but they are also clearly visible;

- The scale effect for the friction component is larger than for the pressure component for both $C_{X}$ and $C_{Y}$. The ratio of the friction component to the pressure component does not remain constant between model and full scale;

- Having in mind that the pressure component of the calculated coefficients, except for $C_{X}$ at 180 degrees, is larger than the friction component, it is not straightforward to apply the extrapolation techniques used for ship resistance based on the form-factor hypothesis, see for instance [17].

Table 6: LNG carrier: difference $\Delta$ between model and full-scale values with comparison uncertainty $U_{c o m p}$. Contribution of pressure $(\mathrm{P})$ and friction $(\mathrm{F})$ to the total force. In green and red the results where $U_{c o m p} \leq \Delta$ and $U_{c o m p}>\Delta$, respectively.

\begin{tabular}{|c|c|c|c|c|c|c|c|c|c|c|c|c|c|c|}
\hline \multirow{3}{*}{$\begin{array}{l}\text { Angle } \\
\text { [deg] }\end{array}$} & \multicolumn{6}{|c|}{ FS versus MS } & \multicolumn{4}{|c|}{ Model scale } & \multicolumn{4}{|c|}{ Full scale } \\
\hline & \multicolumn{2}{|c|}{$C_{X}$} & \multicolumn{2}{|c|}{$C_{Y}$} & \multicolumn{2}{|c|}{$C_{M}$} & \multicolumn{2}{|c|}{$C_{X}$} & \multicolumn{2}{|c|}{$C_{Y}$} & \multicolumn{2}{|c|}{$C_{X}$} & \multicolumn{2}{|c|}{$C_{Y}$} \\
\hline & $\Delta$ & $U_{\text {comp }}$ & $\Delta$ & $U_{\text {comp }}$ & $\Delta$ & $U_{\text {comp }}$ & $\mathrm{P}$ & $\mathrm{F}$ & $\mathrm{P}$ & $\mathrm{F}$ & $\mathrm{P}$ & $\mathrm{F}$ & $\mathrm{P}$ & $\mathrm{F}$ \\
\hline 180 & $-42 \%$ & $26 \%$ & - & - & - & - & $14 \%$ & $86 \%$ & - & - & $14 \%$ & $86 \%$ & - & - \\
\hline 140 & $-16 \%$ & $96 \%$ & $-7.8 \%$ & $2.5 \%$ & $-29 \%$ & $13 \%$ & $74 \%$ & $26 \%$ & $98 \%$ & $2 \%$ & $57 \%$ & $43 \%$ & $99 \%$ & $1 \%$ \\
\hline 90 & - & - & $-3.2 \%$ & $16 \%$ & - & - & - & - & $99 \%$ & $1 \%$ & - & - & $99 \%$ & $1 \%$ \\
\hline
\end{tabular}

\section{RESULTS FOR SEMI-SUBMERSIBLE}

\subsection{Iterative convergence}

For the semi-submersible the level and speed of the iterative convergence are also dependent on the angle, grid resolution and scale of the calculation. Good convergence is often obtained, i.e. a decrease in residuals of more than 5 orders is achieved as illustrated in Figure 7 . However, it also occurs that the iterative convergence stagnates at 4 orders. In general, full-scale calculations are more difficult to converge than for model scale, and for the same inflow angle one order less is obtained. For all cases here presented, the force coefficients become constant after a few hundred iterations and no oscillations are seen. Compared to the LNG carrier the convergence for the semi-submersible is slower and more difficult to obtain due to the unsteadiness of the flow and complex flow with large separated flow regions.

\subsection{Numerical uncertainties}

In Table 7 the results for the semi-submersible without thrusters are presented using subsequently refined grids for model and full scale, all for the angles 180, 150 and 90 degrees. It can be observed that the force coefficients converge when using finer grids for all angles both for model and full scale. Also, for all cases the fine grid results are at most $4 \%$ different from the results on the very fine grid.

The numerical uncertainties $U_{\phi}$ presented in Table 7 show that for model scale the uncertainties are small except for the $C_{X}$ coefficient for 180 degrees and for the $C_{M}$ coefficient for 150 degrees. For 180 

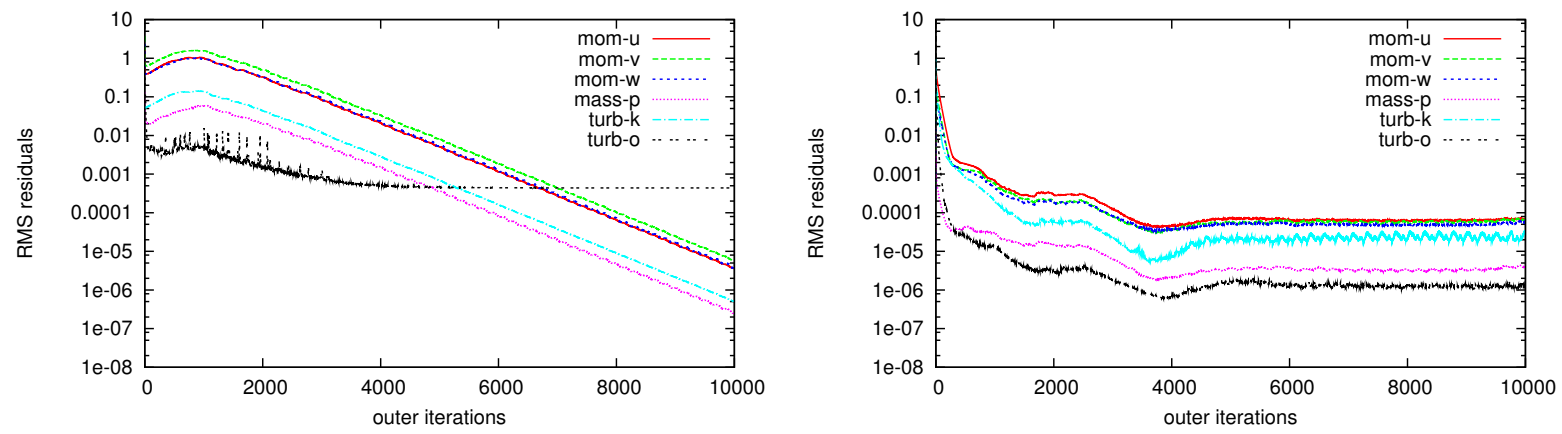

Figure 7: Semi-submersible: examples of iterative convergence for model scale at 90 degrees (left) and full scale at 180 degrees (right).
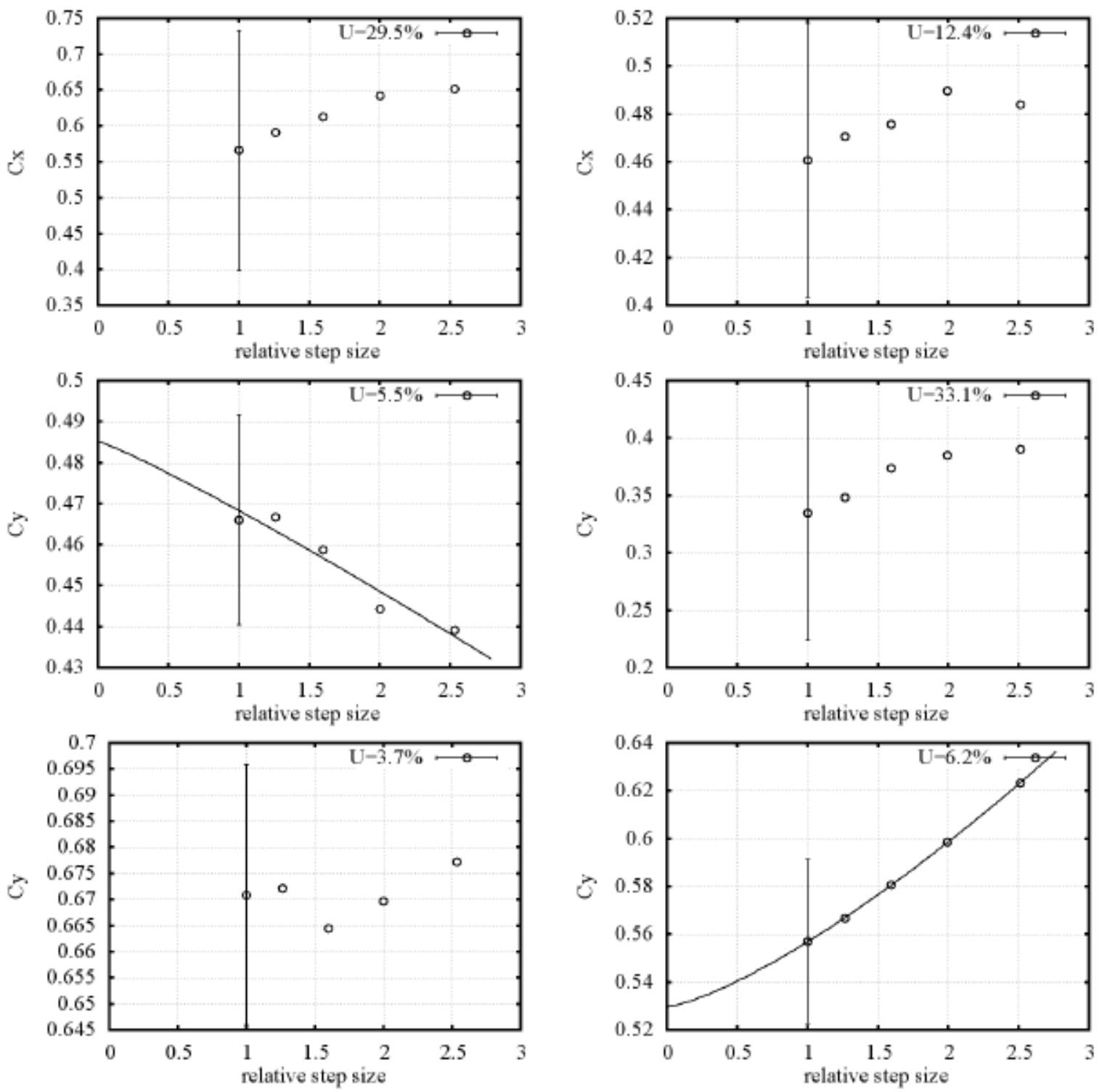

Figure 8: Semi-submersible: examples of numerical uncertainty analysis for $C_{X}$ and $C_{Y}$ coefficient. (left) Model scale (right) Full scale. 
degrees the drag force is very small, leading to a higher uncertainty similar to as found for the LNG carrier. The moment on the semi-submersible is sensitive to the precise location of the flow separation. When refining the grid this location changes slightly leading to a change in the moment. The numerical uncertainties for the full-scale calculations are higher than for model scale.

Table 7: Semi-submersible without thrusters: results for model and full-scale current at 180, 150 and 90 degrees obtained using grids with different resolution. Between brackets the difference compared to the result on the finest grid is presented. The numerical uncertainty $U_{\phi}$ for the results of each coefficient for the finest grid is also presented.

\begin{tabular}{|c|c|c|c|c|c|c|}
\hline & \multirow{2}{*}{ Grid } & 180 degrees & \multicolumn{3}{|c|}{150 degrees } & 90 degrees \\
\hline & & $C_{X}^{*}$ & $C_{X}^{*}$ & $C_{Y}^{*}$ & $C_{M}^{*}$ & $C_{Y}^{*}$ \\
\hline \multirow{6}{*}{ 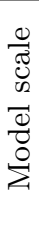 } & very coarse & $0.651(+14.8 \%)$ & $0.810(+3.1 \%)$ & $0.439(-5.8 \%)$ & $0.725(+28.9 \%)$ & $0.677(+0.9 \%)$ \\
\hline & coarse & $0.642(+13.2 \%)$ & $0.799(+1.7 \%)$ & $0.444(-4.7 \%)$ & $0.693(+23.1 \%$ & $0.670(-0.1 \%)$ \\
\hline & medium & $0.612(+7.9 \%)$ & $0.787(+0.1 \%)$ & $0.459(-1.5 \%)$ & $0.614(+9.1 \%)$ & $0.664(-1.0 \%)$ \\
\hline & fine & $0.590(+4.1 \%)$ & $0.786(0.0 \%)$ & $0.467(+0.2 \%)$ & $0.565(+0.4 \%)$ & $0.672(+0.1 \%)$ \\
\hline & very fine & $0.566(0.0 \%)$ & $0.786(0.0 \%)$ & $0.466(0.0 \%)$ & $0.563(0.0 \%)$ & $0.671(0.0 \%)$ \\
\hline & $U_{\phi}$ & $29.5 \%$ & $2.2 \%$ & $5.5 \%$ & $31.6 \%$ & $3.7 \%$ \\
\hline \multirow{6}{*}{ 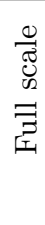 } & very coarse & $0.484(+5.0 \%)$ & $0.782(+8.0 \%)$ & $0.390(+16.8 \%)$ & $0.946(+35.4 \%)$ & $0.623(+11.8 \%)$ \\
\hline & coarse & $0.490(+6.3 \%)$ & $0.771(+6.6 \%)$ & $0.385(+15.3 \%)$ & $0.890(+27.3 \%)$ & $0.598(+7.5 \%)$ \\
\hline & medium & $0.476(+3.3 \%)$ & $0.748(+3.3 \%)$ & $0.373(+12.0 \%)$ & $0.735(+5.1 \%)$ & $0.580(+4.1 \%)$ \\
\hline & fine & $0.471(+2.2 \%)$ & $0.732(+1.1 \%)$ & $0.348(+4.2 \%)$ & $0.712(+1.9 \%)$ & $0.567(+1.8 \%)$ \\
\hline & very fine & $0.461(0.0 \%)$ & $0.724(0.0 \%)$ & $0.334(0.0 \%)$ & $0.699(0.0 \%)$ & $0.557(0.0 \%)$ \\
\hline & $\overline{U_{\phi}}$ & $12.4 \%$ & $21.8 \%$ & $33.1 \%$ & $19.7 \%$ & $6.2 \%$ \\
\hline
\end{tabular}

\subsection{Validation with model-scale experiments}

In the wind-tunnel experiments eight thrusters were modelled under the hull of the semi-submersible. For validation purposes it would have been better to test and calculate the bare hull of the semisubmersible. In [5] the effect of modeling the thrusters has been investigated and it was concluded that for certain current headings the thrusters have a significant effect on the calculated results. Therefore, for the comparison between the CFD and wind tunnel results, following [5], the thrusters have been taken into account in the CFD calculations presented in Figure 9 and Table 8. However, in the wind tunnel simple rings were used for the thrusters. In the CFD calculations the exact geometry of the thrusters has been taken into account but the thrusters have been closed. This leads to an additional uncertainty when comparing the results between wind tunnel and CFD, which can be taken into account through the parameter uncertainty $U_{i n p}$ in Equation 1. We assume that this uncertainty due to the thrusters is 4\%. Furthermore, as explained in Section 3.2, the pontoons in the wind tunnel were $4.5 \%$ shorter than in the CFD calculations. This leads to approximately $1 \%$ larger drag forces and $3 \%$ larger side forces in the CFD calculations. Therefore, we assume that the total value for the parameter uncertainty $U_{\text {inp }}$ is equal to $8 \%$.

From Figure 9 it appears that good agreement between the wind tunnel and CFD is obtained. For the angles with larger forces, i.e. the range 180 to 130 degrees for $C_{X}^{*}$ and the range 140 to 90 degrees for $C_{Y}^{*}$, the CFD results are validated and within $12 \%$ or lower from the wind tunnel results. However, for $C_{X}^{*}$ the validation uncertainty $U_{v a l}$ is large and both the numerical and experimental uncertainty should be decreased.

Table 8: Semi-submersible with thrusters: comparison difference $E$ between model-scale CFD results and experimental values and validation uncertainty $U_{v a l}$. In green the validated results and in red the non-validated results.

\begin{tabular}{|c|c|cccccccccc|}
\hline & \multirow{2}{*}{ Coefficient } & \multicolumn{10}{|c|}{ Angle [deg] } \\
\cline { 3 - 11 } & & 180 & 170 & 160 & 150 & 140 & 130 & 120 & 110 & 100 & 90 \\
\hline \multirow{2}{*}{$E$} & $C_{X}^{*}$ & $+9 \%$ & $+16 \%$ & $+4 \%$ & $-2 \%$ & $-12 \%$ & $-9 \%$ & $-35 \%$ & $-46 \%$ & $-73 \%$ & - \\
& $C_{Y}^{*}$ & - & $-27 \%$ & $-27 \%$ & $-15 \%$ & $-8 \%$ & $-12 \%$ & $-10 \%$ & $-11 \%$ & $-11 \%$ & $-11 \%$ \\
\hline \multirow{2}{*}{$U_{\text {val }}$} & $C_{X}^{*}$ & $35 \%$ & $37 \%$ & $33 \%$ & $13 \%$ & $29 \%$ & $30 \%$ & $22 \%$ & $19 \%$ & $13 \%$ & $10 \%$ \\
& $C_{Y}^{*}$ & $10 \%$ & $12 \%$ & $12 \%$ & $13 \%$ & $13 \%$ & $13 \%$ & $13 \%$ & $13 \%$ & $13 \%$ & $13 \%$ \\
\hline
\end{tabular}



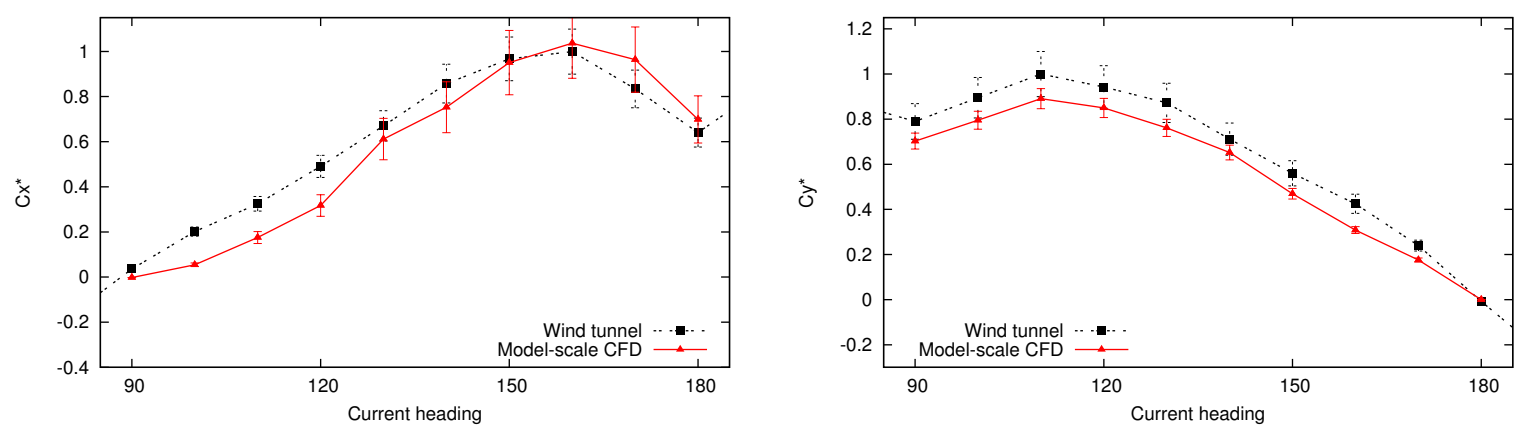

Figure 9: Semi-submersible with thrusters: comparison of CFD results with experimental results.

\subsection{Scale effects}

Scale effects are determined for the semi-submersible without thrusters using the fine grid for both model and full scale. It can be observed in Figure 10 and Table 9 that for the angles with larger $C_{X}^{*}$ value, i.e. the angles from 180 to 120 degrees, the difference between model scale and full scale is approximately 6-30\%.

For the angles with larger $C_{Y}^{*}$ values, i.e. the angles from 160 to 90 degrees, the difference is larger: 6-43\%. It should be noted that the pressure component of the force for the semi is highly dominant, i.e. more than $90 \%$ of the total force originates from the pressure distribution on the semi, even for the $C_{X}^{*}$ at 180 degrees. This is due to the blunt-body shape of the structure. The ratio between the pressure and friction component changes slightly from model to full scale.

When considering the difference $\Delta$ between model and full-scale results and the comparison uncertainty $U_{\text {comp }}=\sqrt{U_{\phi, M S}^{2}+U_{\phi, F S}^{2}}$, as presented in Table 9, we conclude that the scale effects have been captured correctly for $C_{Y}^{*}$ since the difference $\Delta$ is larger than the comparison uncertainty. For $C_{X}^{*}$ and $C_{M}^{*}$ the numerical uncertainty of the full-scale results are large indicating that one should be careful to draw strong conclusions based on these full-scale results.

Table 9: Semi-submersible without thrusters: difference between model and full-scale values and contribution of pressure (P) and friction (F) to the total force. Results obtained on fine grid. In green and red the results where $U_{\text {comp }} \leq \Delta$ and $U_{\text {comp }}>\Delta$, respectively.

\begin{tabular}{|c|c|c|c|c|c|c|c|c|c|c|c|c|c|c|}
\hline \multirow{3}{*}{$\begin{array}{c}\text { Angle } \\
\text { [deg] }\end{array}$} & \multicolumn{6}{|c|}{ FS versus MS } & \multicolumn{4}{|c|}{ Model scale } & \multicolumn{4}{|c|}{ Full scale } \\
\hline & \multicolumn{2}{|c|}{$C_{X}^{*}$} & \multicolumn{2}{|c|}{$C_{Y}^{*}$} & \multicolumn{2}{|c|}{$C_{M}^{*}$} & \multicolumn{2}{|c|}{$C_{X}^{*}$} & \multicolumn{2}{|c|}{$C_{Y}^{*}$} & \multicolumn{2}{|c|}{$C_{X}^{*}$} & \multicolumn{2}{|c|}{$C_{Y}^{*}$} \\
\hline & $\Delta$ & $U_{\text {comp }}$ & $\Delta$ & $U_{\text {comp }}$ & $\Delta$ & $U_{\text {comp }}$ & $\mathrm{P}$ & $\mathrm{F}$ & $\mathrm{P}$ & $\mathrm{F}$ & $\mathrm{P}$ & $\mathrm{F}$ & $\mathrm{P}$ & $\mathrm{F}$ \\
\hline 180 & $-20 \%$ & $32 \%$ & - & - & & - & $90 \%$ & $10 \%$ & & - & $95 \%$ & $5 \%$ & & \\
\hline 150 & $-9 \%$ & $22 \%$ & $-36 \%$ & $33 \%$ & $+20 \%$ & $37 \%$ & $92 \%$ & $8 \%$ & $98 \%$ & $2 \%$ & $97 \%$ & $3 \%$ & $99 \%$ & $1 \%$ \\
\hline 90 & - & - & $-19 \%$ & $7 \%$ & - & - & - & - & $98 \%$ & $2 \%$ & - & - & $99 \%$ & $1 \%$ \\
\hline
\end{tabular}

\subsection{Unsteady calculations}

To investigate the change in forces due to unsteady effects preliminary unsteady URANS calculations are carried out. These calculations require large CPU time, and for practical applications they are usually not performed. The results here presented are for both model and full scale for current headings 180 and 150 degrees. For 180 degrees the flow is very unsteady due to vortices being shed from the first columns which interfere with the columns located in the wake. At 150 degrees significant flow separation occurs characterized by large, steady, coherent vortices along the keel of the semi-submersible resulting in a very steady flow pattern. Different grid resolution and time step sizes are used: the medium, fine and very fine grid together with time step sizes equal to $T_{\text {ref }} / 50, T_{\text {ref }} / 100$ and $T_{\text {ref }} / 200$ with $T_{\text {ref }}$ the reference time here defined by $D / U_{\text {ref. }}$. In the near future the same investigations for heading 90 degrees will be carried out.

During the unsteady calculations the level of iterative convergence is aimed to be lower than $10^{-4}$ as visible in Figure 11(a). The number of iterations per time step have an influence on the level of 

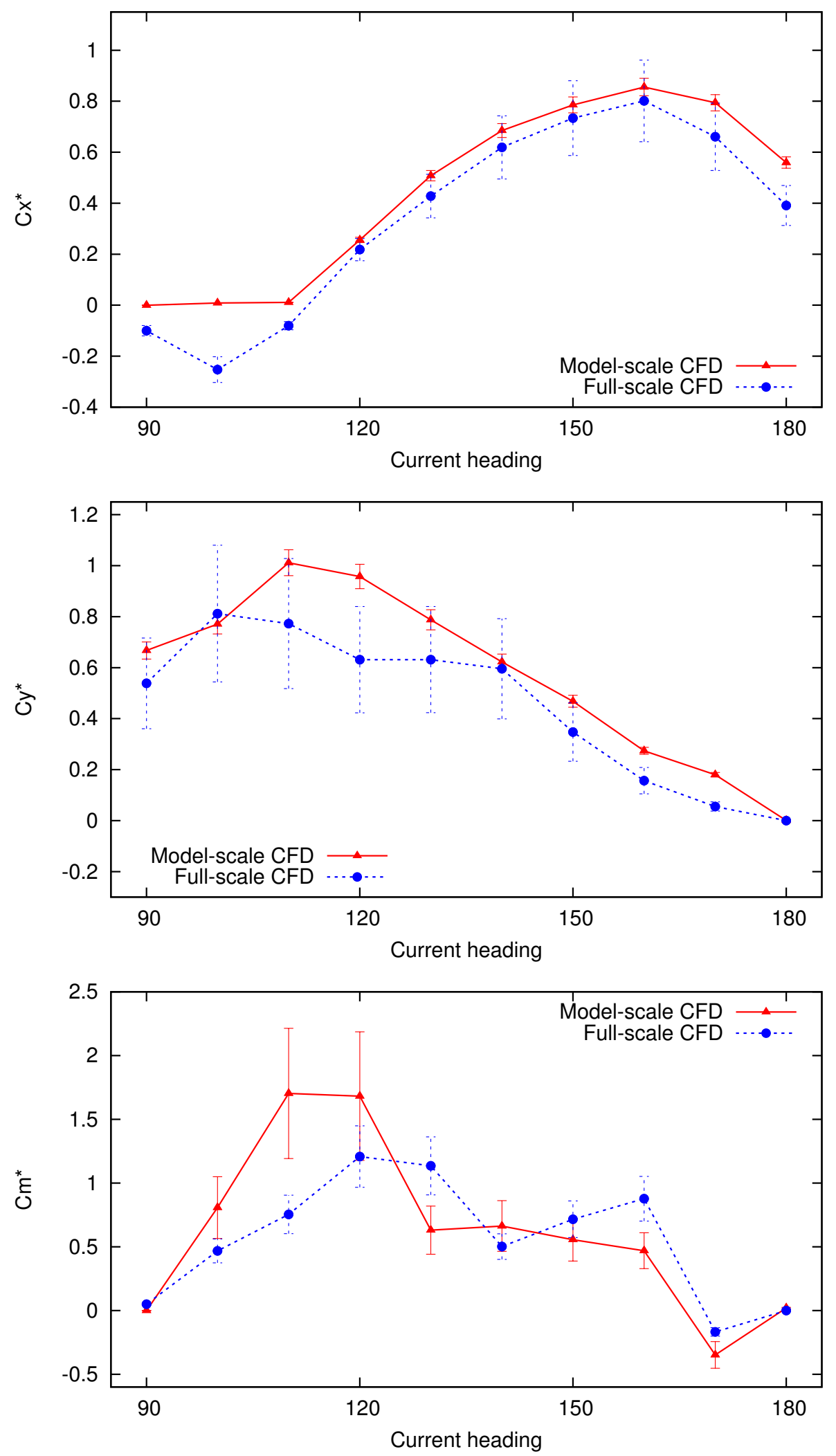

Figure 10: Semi-submersible without thrusters: model and full-scale current coefficients. 


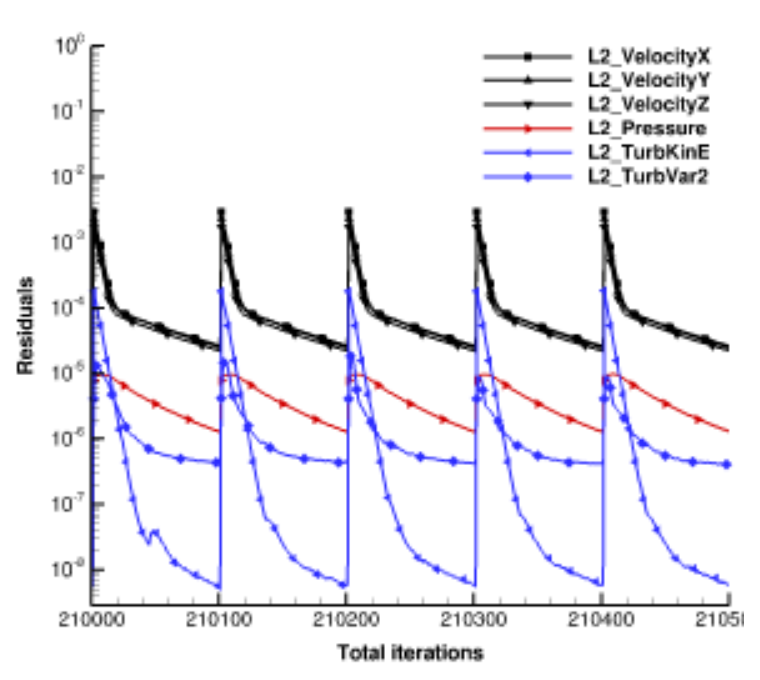

(a)

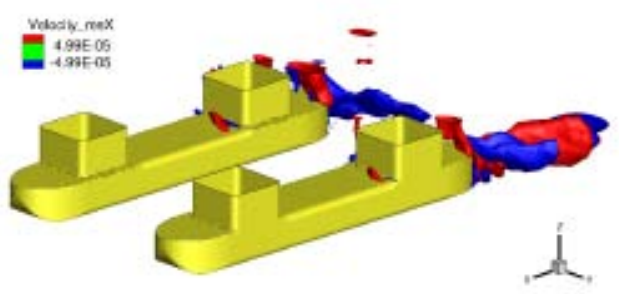

(b)

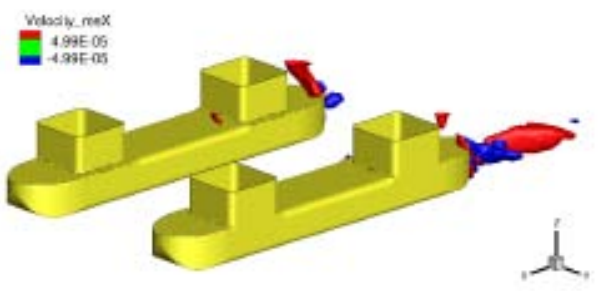

(c)

Figure 11: Semi-submersible without thrusters: unsteady model-scale calculations at 180 degrees using the fine grid and $\Delta t=T_{\text {ref }} / 50$. (a) Residuals during time steps. Highest residuals in the flow field at the last iteration of a certain time step using (b) 50 or (c) 100 iterations per time step.

convergence: the more iterations per time step are taken the better the level of convergence is. The velocity variables have the highest level of residuals and these are located in the wake behind the semisubmersible as visible in Figures 11(b) and (c). It can also be observed that when using 100 iterations per time step instead of 50, the regions with higher residuals decrease in size. For model scale using a different number of iterations per time step, i.e. aiming at a better convergence level at each time step, does not result in significant differences in the calculated unsteady forces, provided that the convergence level at each time step is lower than $10^{-4}$. For full scale this convergence level is more difficult to obtain. As a result the time traces of the calculated forces differ significantly when using a different grid resolution or time step size. Therefore, at full scale more outer loops need to be taken to satisfy the convergence level of $10^{-4}$. However, this also results in longer calculation times.

For model scale the calculated time-dependent forces become nicely periodic as illustrated in Figure 12. Using a different grid resolution and time step size results in very similar time-dependent forces, only small differences of less than $2 \%$ are found for the maximum and minimum values of the forces. For full scale the time traces of the calculated forces significantly differ when using a different grid resolution or time step size. This is caused by the convergence problems as described above and should be further investigated.

In Table 10 the steady and the unsteady average results obtained with CFD for model and full scale are compared with the results from the wind tunnel. Note that for a proper comparison the numerical uncertainty $U_{p h i}$ should be obtained for the unsteady calculations. It can be observed that the unsteady average is almost equal to the steady-state result for all cases. The model-scale values obtained with CFD are less than $11 \%$ different from the wind tunnel. The full-scale CFD values are $7-29 \%$ different from the model-scale CFD values. Contrary to the obtained results in [1] we do not find significant unsteady effects on the calculated average force coefficients. However, only two current angles have been investigated in this paper and only one angle in [1]. One major issue is the iterative convergence for unsteady calculations, especially at full scale. The other point may be that the grid topology and its refinement here used might not be adequate for unsteady calculations, where highly refined grids are needed in the shear-layers and in particular in the wakes of all structure components. Furthermore, the numerical uncertainty $U_{\phi}$ should be obtained for these unsteady calculations using different grid resolution and time step size.

Therefore, we recommend to investigate the unsteady effects further despite the long calculation times required. 


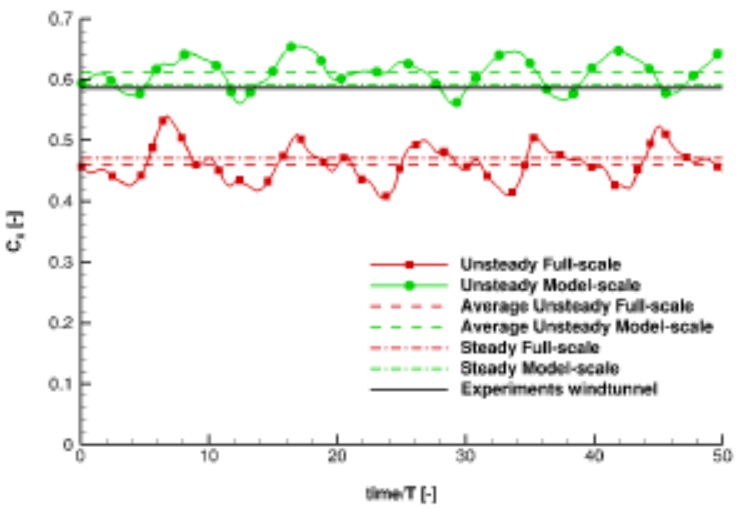

(a) $C_{X}^{*}$

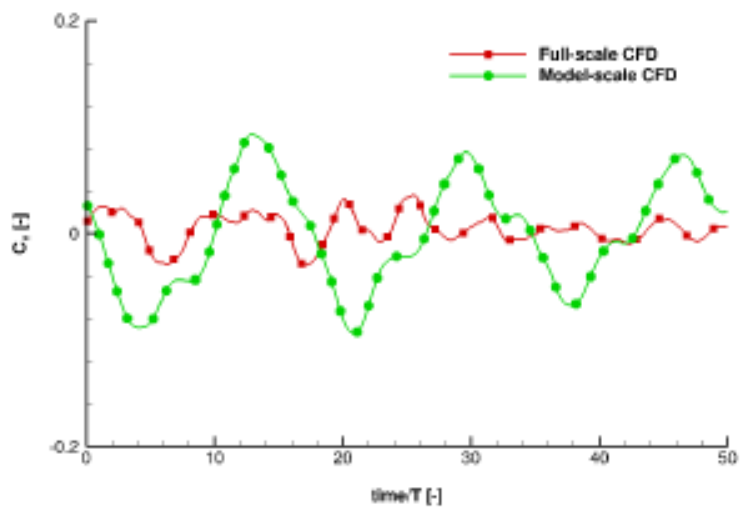

(b) $C_{Y}^{*}$

Figure 12: Semi-submersible without thrusters: unsteady model and full-scale force coefficients at 180 degrees.

Table 10: Semi-submersible without thrusters: results for current coefficients at 180 and 150 degrees. The steady results and the unsteady average are compared with the results from the wind tunnel. The full-scale values obtained with CFD are compared to the model-scale CFD results.

\begin{tabular}{|c|c|ccc|}
\hline & 180 degrees & \multicolumn{3}{|c|}{150 degrees } \\
& $C_{X}^{*}$ & $C_{X}^{*}$ & $C_{Y}^{*}$ & $C_{M}^{*}$ \\
\hline Windtunnel & - & - & - & - \\
Steady MS & $+1 \%$ & $-11 \%$ & $-9 \%$ & $+3 \%$ \\
Unsteady MS & $+4 \%$ & $-11 \%$ & $-9 \%$ & $+6 \%$ \\
\hline Steady FS & $-20 \%$ & $-7 \%$ & $-26 \%$ & $+29 \%$ \\
Unsteady FS & $-22 \%$ & $-7 \%$ & $-24 \%$ & $+22 \%$ \\
\hline
\end{tabular}

\section{CONCLUSIONS AND FUTURE WORK}

In this paper, CFD calculations for current loads on an LNG carrier and a semi-submersible are presented, both for model and full-scale situations, for current angles ranging from 180 to 0 degrees. MARIN's in-house URANS code ReFRESCO is used. Numerical studies are carried out concerning iterative convergence and grid refinement. In total, more than 100 calculations have been performed. Detailed verification analysis is carried out using modern techniques, and numerical uncertainties are calculated. Afterwards, quantitative validation for model-scale Reynolds number is done. Scale effects on the current coefficients are investigated, having in mind the estimated numerical uncertainties, and unsteady effects are briefly studied.

Good iterative convergence is obtained in most calculations, i.e. a decrease in residuals of more than 5 orders is achieved. The level and speed of the iterative convergence is dependent on the current angle, grid resolution and scale of the calculation. Nicely streamlined flows are easier to solve than flows with large separated flow regions. Full-scale calculations are more difficult to converge than for model scale and for the same flow angle one order less is obtained. For unsteady model-scale calculations a better iterative convergence level does not result in significant changes in the calculated unsteady forces, provided that the convergence level at each time step is lower than $10^{-4}$. For full scale this convergence level is more difficult to obtain and more iterations per time step should be taken to satisfy this convergence level. However, the authors emphasize that an adequate absolute value for iterative convergence is very much dependent on the employed linear solvers, residual normalization and numerical tool.

The sensitivity to grid resolution at model and full scale has been investigated for both cases using five consecutively refined grids and for 3 current headings. The differences in the solution between two consecutive refinements converge for all cases. The fine grid results, i.e. 3 million cells for the LNG carrier and 7 million cells for the semi-submersible, are at most $4 \%$ different from the results on the very-fine grid with 6 million cells for the LNG carrier and 20 million cells for the semi-submersible. However, this does not mean that the numerical uncertainties are low. The numerical uncertainties are larger for angles 
with small values of the loads, which is also expected for the experimental results. In some cases, such as $C_{X}$ for 180 degrees current heading at model-scale, the numerical uncertainty value is too large, $29.5 \%$. In order to further decrease the numerical uncertainties, better iterative convergence should be achieved and even finer grids should be used. In general, for full-scale situations the numerical uncertainties are higher. Also, for full-scale situations no wall-functions should be used, since this adds an additional modelling inaccuracy, and possibility of numerical scattering due to different boundary conditions for different grids.

Comparison with experiments shows that ReFRESCO provides good quantitative prediction of the current loads at model scale. Taking into account the numerical and experimental uncertainties, it is found that for angles with larger forces the CFD results are validated with $15 \%$ of uncertainty. Nevertheless, for the semi-submersible, for some validated situations, the validation uncertainties are too large due to the numerical uncertainties, but also due to the large experimental and input-parameters uncertainties. This should be further investigated.

To determine scale effects the numerical uncertainties must be considered in order to prevent wrong conclusions drawn on basis of numerical differences rather than on physical differences. When the difference between model and full-scale results is smaller than the comparison uncertainty these values should be considered with care. For the full-scale results larger numerical uncertainties are found than for model scale and for absolute values for scale effects this uncertainty should be improved. For the LNG carrier significant scale effects, i.e. more than $40 \%$, have been obtained for current angles where the friction component is dominant. For these cases the numerical uncertainty is relatively low. For the other current angles differences of $8-30 \%$ between model and full scale can be observed, but here larger uncertainties are found. For the semi-submersible the numerical uncertainties for the full-scale results are larger than for the LNG carrier. For the semi-submersible the pressure component of the force is highly dominant, i.e. larger than $90 \%$ of the total force. On average the full-scale current coefficients are $20 \%$ lower than at model scale, but larger differences for a number of angles can be observed. For both the semi-submersible and the LNG carrier it is found that the ratio between the pressure contribution and friction contribution to the force does not remain constant comparing model scale to full scale. For the angles where the pressure component is larger than the friction component, it is not straightforward to apply extrapolation methods as used for ship resistance.

Lastly, a preliminary study into the unsteady effects on the current loads has been carried out. These calculations require much CPU time and are therefore only presented for 180 and 150 degrees current heading. Contrary to the obtained results from [1] we do not find significant unsteady effects on the average of the calculated force coefficients. However, only two current angles have been investigated in this paper and only one angle in [1]. More calculations for different headings should be carried out before a valid conclusion on unsteady effects can be drawn. Unsteady problems can be found in many offshore applications such as Vortex Induced Motions (VIM), illustrating the importance to accurately calculate the unsteady flow. Therefore, we recommend to further investigate the unsteady effects despite the long calculation times required. 


\section{REFERENCES}

[1] G. Vaz, O. Waals, F. Fathi, H. Ottens, T. Le Souef, and K. Kwong. Current Affairs - Model Tests, Semi-Empirical Predictions and CFD Computations for Current Coefficients of Semi-Submersibles. In Proceedings of OMAE2009, Honolulu, Hawaii, USA, June 2009.

[2] L. Eça, G. Vaz, and M. Hoekstra. A Verification and Validation Exercise for the Flow Over a Backward Facing Step. In Proceedings of ECCOMAS-CFD2010, Lisbon, Portugal, June 2010.

[3] G. Vaz, F. Jaouen, and M. Hoekstra. Free-Surface Viscous Flow Computations. Validation of URANS Code FreSCo. In Proceedings of OMAE2009, Honolulu, Hawaii, USA, June 2009.

[4] F. Fathi, C.M. Klaij, and A. Koop. Predicting Loads on a LNG Carrier with CFD. In Proceedings of OMAE2010, Shanghai, China, June 2010.

[5] A. Koop and A. Bereznitski. Model-Scale and Full-Scale CFD Calculations for Current Loads on Semi-Submersible. In Proceedings of OMAE2011, Rotterdam, the Netherlands, June 2011.

[6] Oil Companies International Marine Forum. Prediction of Wind and Current Loads on VLCCs. $2^{\text {nd }}$ edition, 1994.

[7] O.J. Waals. Current Force Measurements on a 135,000 $\mathrm{m}^{3}$ and 224,000 $\mathrm{m}^{3}$ LNG Carrier - HAWAII JIP Current Coefficients. Technical Report 19436-1-BT, MARIN, 2007.

[8] http://www.forcetechnology.dk.

[9] http://www.gridpro.com/.

[10] A. Koop, C.M. Klaij, and G. Vaz. Predicting Wind Shielding for FPSO Tandem Offloading using CFD. In Proceedings of OMAE2010, Shanghai, China, June 2010.

[11] G. Vaz, S.L. Toxopeus, and S. Holmes. Calculation of Manoeuvring Forces on Submarines Using Two Viscous-Flow Solvers. In Proceedings of OMAE2010, Shanghai, China, June 2010.

[12] F. Menter. Two-equation Eddy Viscosity Turbulence Models for Engineering Applications. AIAA Journal, 32:1598-1605, 1994.

[13] American Institute For Aeronautics and Astronautics. Guide for Verification and Validation of Computational Fluid Dynamics Simulations. Technical Report AIAA-G-077-1998, AIAA, 1998.

[14] American Society of Mechanical Engineers. ASME Guide on Verification and Validation in Computational Fluid Dynamics and Heat Transfer. Technical Report ASME Committee PTC-61, ANSI Standard V\&V-20, 2008.

[15] D. Rijpkema and G. Vaz. Viscous Flow Computations on Propulsors: Verification, Validation and Scale Effects. In Proceedings of RINA-CFD2011, London, UK., March 2011.

[16] S. L. Toxopeus. Practical application of viscous-flow calculations for the simulation of manoeuvring ships. PhD thesis, Delft University of Technology, Faculty Mechanical, Maritime and Materials Engineering, May 2011.

[17] H.C. Raven, A. van der Ploeg, A.R. Starke, and L. Eça. Towards A CFD-Based Prediction Of Ship Performance. Progress In Predicting Full-Scale Resistance And Scale Effects. Proceedings of RINA-CFD-2008. London, UK, August 2008. 